\title{
Acute Cardiac Tamponade in a 77-year-old Italian Woman with Erdheim-Chester Disease
}

\author{
Maria Kyriakopoulou, Guy Decaux, Mike El Mourad, Ruben Casado-Arroyo \\ Cardiology Department, Cliniques Universitaires Erasme, Bruxelles, Belgium
}

Received: 20/06/2016

Accepted: 25/08/2016

Published: $19 / 09 / 2016$

How to cite this article: Kyriakopoulou M, Decaux G, El Mourad M, Casado-Arroyo R. Acute cardiac tamponade in a 77-year-old italian woman with Erdheim-Chester disease. EJCRIM 2016;3:doi:10.12890/2016_000451.

Conflicts of Interests: The Authors declare that there are no competing interests.

This article is licensed under a Commons Attribution Non-Commercial 4.0 License

\section{ABSTRACT}

Erdheim-Chester disease (ECD) is a non-Langerhans' histiocytosisand a veryrare multisystemic disease of unknown aetiology, with skeletal involvement of the long bones and in more than $50 \%$ of cases with extraskeletal involvement. The disease was described in 1930 by the anatomopathologist Jakob Erdheim and his student William Chester. More than 500 cases have since been reported. We report the case of a 77-year-old Italian woman with ECD who was admitted to hospital for acute cardiac tamponade. The patient presented with simultaneous cutaneous, retro-orbital, skeletal, cerebral and cardiovascular manifestations and was successfully treated with corticosteroids followed by interferon.

\section{LEARNING POINTS}

- Erdheim-Chester disease (ECD) is a non-Langerhans' histiocytosisand a very rare multisystemic disease that is thought to be associated with cytokine disturbances.

- ECD has a variable prognosis but is poorer in those with heart involvement.

- First-line treatment involves the administration of interferon alpha.

\section{KEYWORDS}

Erdheim-Chester disease, tamponade, BRAF, interferon alpha

\section{INTRODUCTION}

Erdheim Chester disease (ECD) typically presents in 40-60-year-old adults and has a male predominance. The natural history of the disease varies from asymptomatic to multisystemic, life-threatening forms. The diagnosis of ECD is based on histological and radiological criteria. The characteristic histological image is a xanthomatous or xanthogranulomatous infiltration of tissues by spumous histiocytes, surrounded by fibrosis. ECD can be distinguished from Langerhans' cell histiocytosis by the immunohistological and microscopic characteristics of the histiocytes. ECD patients are positive for CD68 and negative for CD1a and have no Birbeck granules. Extra-skeletal involvement may include the skin, kidneys, retro-orbital tissue, central nervous system, heart, lungs, liver, thyroid, testicles, breast or ganglions ${ }^{[1]}$. 


\section{CASE REPORT}

A 77-year-old Italian woman with a medical history of diplopia and glaucoma was admitted to our hospital for dyspnoea, orthopnoea and lower extremity oedema.She had a 4-month history of bilateral exophthalmos, orbital xanthogranuloma and fatigue (Fig. 1). Her medical history included Barrett's oesophagus, hepatic steatosis, vesicular lithiasis, haemorrhoids, hypovitaminosis D, spine fractures, cataract, bladder prolapse, sacrocolpopexy, hysterectomy and ovariectomy. She was allergic to penicillin. She was taking a collyrium with carteolol once per day, calcium once per day and vitamin D once per week. She was a lifelong non-smoker, did not consume alcohol, had not travelled abroad recently and had never used illicit drugs.

On physical examination she had a temperature of $36^{\circ} \mathrm{C}$, heart rate of 85 beats/minuteand blood pressure of $125 / 75 \mathrm{mmHg}$. Shewas dyspnoeic (NYHA III b) and orthopnoeic. She presented with remarkable bilateral painless exophthalmos, palpebral oedema, orbital xanthogranuloma and jugular turgescence. The cardiovascular examination showed normal S1 and S2 with no added sounds. The respiratory examination showed diminished breath sounds at the bases of the lungs and decreased fremitus. She also had bilateral lower extremity oedema.

Emergent cardiac echography (Fig. 2) revealed a circumferential pericardial effusion with an anterior predominance, compression of the right ventricle, septal shift and variations of $50 \%$ in mitral and aortic flow, all signs of acute cardiac tamponade. Urgent pericardiocentesis was carried out and $1300 \mathrm{ml}$ of serous liquid was removed. The patient was then admitted to the intensive heart unit for further monitoring. The clinical evolution was excellent and on the fifth day she was transferred to the department of internal medicine so diagnostic investigations could be continued.

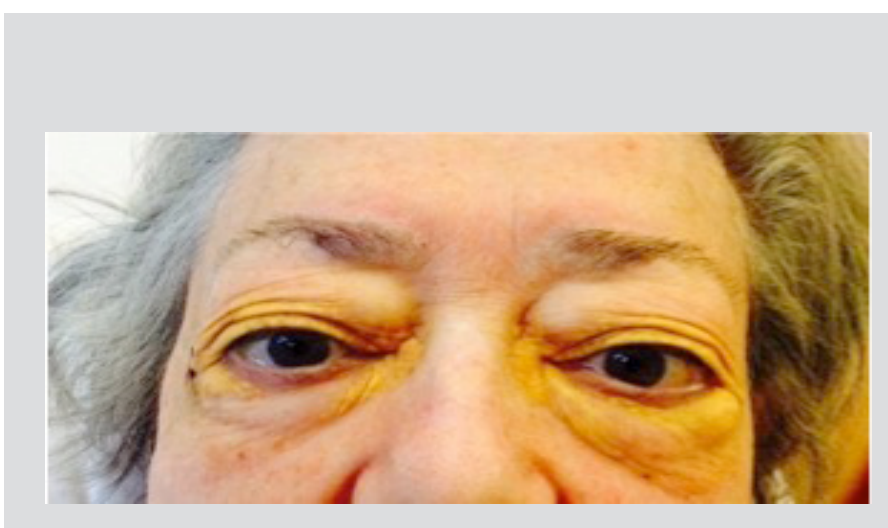

Figure 1. Bilateral exophthalmos and xanthogranuloma

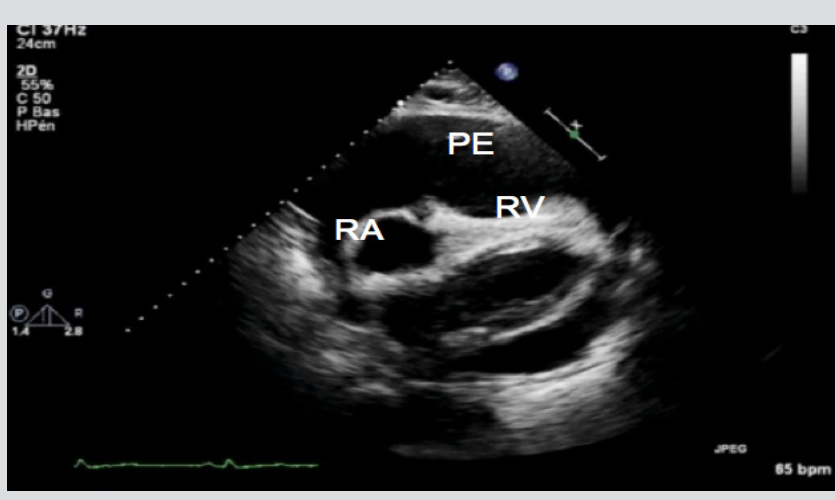

Figure 2. Cardiac echography

( $P E$, pericardial effusion; $R A$, right atrium; $R V$, right ventricle)

Laboratory tests showed lymphopenia $755 / \mathrm{mm}^{3}$ (normal 1340-3950/mm³), elevated CRP 59 mg/l (normal < 10 mg/l), normal renal and liver function, and low albumin $29 \mathrm{~g} / \mathrm{l}$ (normal 32-46 g/l). Chest radiography (Fig. 3) showed cardiomegaly and mild bilateral pleural effusion. ECG showed sinus rhythm with right bundle branch block. The particular phenotype of the patient with bilateral exophthalmos and orbital xanthogranuloma of recent onset with the combination of dyspnoeaand pericardial and pleural effusion suggested that this case was not an ordinary cardiac tamponadeof idiopathic, infectious, autoimmune or metabolic aetiology. We therefore considered multisystemic disease. Analysis of the pericardial liquid showed spumous histiocytes, anti CD 68+, anti CD 1a-and anti S100-.PET CT (Fig. 4) showed orbital, humeral, radial, trochanteric, femoral, colic and pleural FDG uptake. There was also FDG uptake by the thoracic aorta, the pericardium, the right atrioventricular orificeand the left breast. Femoral biopsy showed spumous histiocytes, anti CD 68+, anti CD 1a-, anti S100- and Touton giant cells. There was no BRAF mutation, but mutation of kit M541L was found. Cardiac MRI (Fig. 5) showed pericardial thickening associated with delayed enhancement, delayed enhancement at the atrioventricular orifices, and circumferential non-transmural enhancement of the thoracic aortic wall (at the adventitia, sparing the muscular layer). Cerebral MRI showed pachymeningitis with a multifocal pseudo-tumoural character and bilateral orbital infiltration.

After histological diagnosis of ECD with multi-organ involvement, treatment with intravenous corticosteroids (500 mg methylprednisoloneonce per day) was administered for 3 days followed by subcutaneous interferon alfa-2b ( 3 million units twice per week for 1 week and then 6 million units three timesper week). The patient was discharged from the hospital 2 weeks later. After almost 1 week she visited her doctor and interferon alfa-2b was changed to peginterferon alfa-2a (135 units once per week) with better tolerance but with 
moderate fatigue and a spike in temperature $\left(38^{\circ} \mathrm{C}\right)$ on the day of injection. The patient is still alive 1 year later, and the exophthalmos has partially regressed.

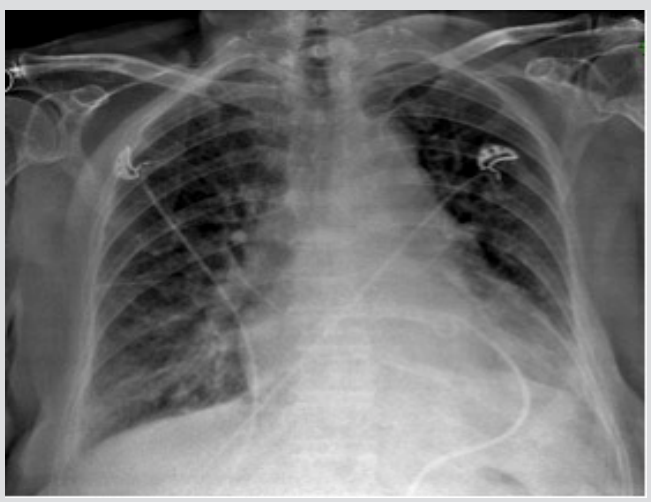

Figure 3. Chest radiogram showing cardiomegaly and mild bilateral pleural effusion

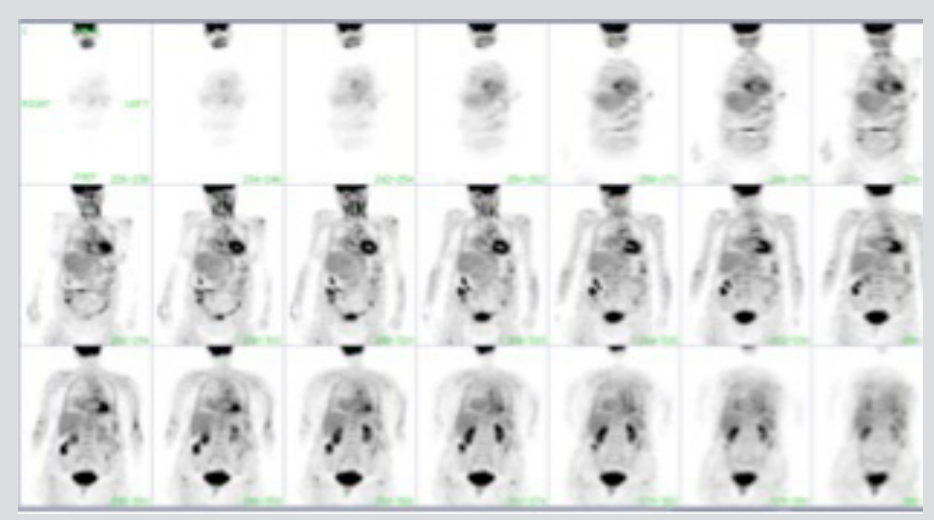

Figure 4. PET CT howing orbital, humeral, radial, trochanteric, femoral, colic and pleural FDG uptakeand FDG uptake by the thoracic aorta, the pericardium, the right atrioventricular orifice and the left breast
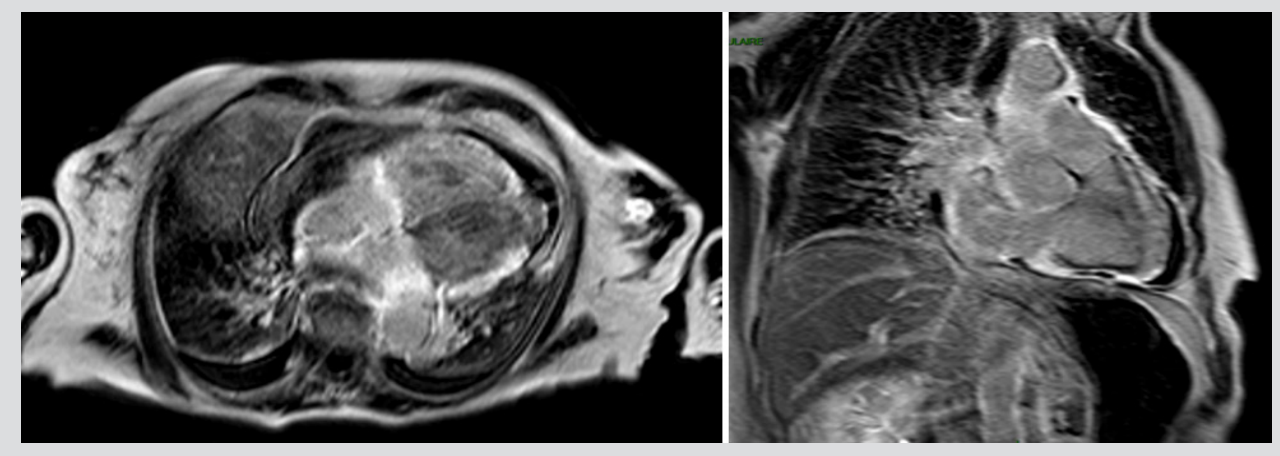

Figure 5. Cardiac MRI showing pericardial thickening associated with delayed enhancement, delayed enhancement at the atrioventricular orifices, and circumferential nontransmural enhancement of the thoracic aortic wall (at the adventitia, sparing the muscular layer)

\section{DISCUSSION}

We described the case of a 77-year-old Italian woman with ECD who was admitted to hospital for acute cardiac tamponade, presented with simultaneous cutaneous, retro-orbital, skeletal, cerebral and cardiovascular manifestations and was successfully treated with corticosteroids followed by interferon. We diagnosed ECD based on clinical, radiological and histological findings.

The aetiology of ECD is unknown but it is thought to be associated with cytokine disturbances. Elevated levels of interferonalfa, interleukin (IL)-7, IL-12, monocyte chemo attractant protein-1 and decreased levels of IL-4 found in ECD patients support an associated systemic immune Th-1 oriented perturbation. Recent findings of mutations in the BRAF proto-oncogene in $>50 \%$ of ECD cases clearly add further complexity to the pathophysiology of $\mathrm{ECD}^{[2,3]}$.

The differential diagnosis of EDC includes Langerhans' cell histiocytosis, Rosai-Dorfman disease, Takayasu vasculitis, Wegener's granulomatosis, primary hypophysitis, chronic recurrent multifocal osteomyelitis, malignancy and sarcoidosis.

There are no consensus guidelines for the treatment of EDC because no large studies have been performed. However, different treatments have been used individually with various levels of success. These include systemic corticosteroids, immunotherapy (interferon) and chemotherapy.

First-line treatment involves the administration of interferon alfafor all forms of ECD with higher doses required on a long-term basis for those with CNS and cardiac involvement. Second-line therapies include anakinra, vemurafenib, imatinib and cladarbine. Salvage therapies include vincristine, vinblastine, cyclophosphamide, methotrexate and doxorubicin. 
Palliative therapies include prednisolone and bisphosphonates. Other treatments used include infliximab, tocilizumab, mycophenolate and stem cell transplantation. Recently, infliximab and vemurafenib have been used with some success; vemurafenib seems to be promising for patients with a BRAFV600 mutation, but new prospective trials are needed ${ }^{[1,2,4,5]}$. It is important to emphasize that while these treatments may control the symptoms and delayevolution of the disease, there is as yet no cure for ECD.

\section{CONCLUSION}

ECD has a variable prognosis which is poorer in those with central nervous system and heart involvement. Before interferon alfa became available, mean survival after diagnosis was 19.2 months. Now, with interferon alfa treatment, the mortality rate is only $26 \%$ and 5 -year survival is $68 \%$. The main cause of death is heart, respiratory or renal failure ${ }^{[2,3]}$.

ECD is a very rare disorder and often difficult to diagnose. It may be months or even years after symptom onset before patients are finally diagnosed and properly treated. Cardiovascular involvement in particular, is common but often asymptomatic and underdiagnosed. Consequently, physicians must be very vigilant when there is clinical suspicion of ECD.

\section{REFERENCES}

1. Mazor RD, Manevich-Mazor M, Shoenfeld Y. Erdheim-Chester disease: a comprehensive review of the literature. Orphanet J Rare Dis 2013;8:137.

2. Arnaud L, Hervier B, Neel A, Hamidou MA, Kahn JE, Wechsler B, et al. CNS involvement and treatment with interferon-alpha are independent prognostic factors in ErdheimChester disease: a multicenter survival analysis of 53 patients. Blood 2011;117:2778-2782.

3. Haroche J, Arnaud L, Amoura Z. Erdheim-Chester disease. Curr Opin Rheumatol 2012;24:53-59.

4. MunozJ, Janku F, Cohen P,Kurzrock R. Erdheim-Chester disease: characteristics and management. Mayo Clin Proc 2014;89:985-996.

5. Haroche J, Cohen-Aubart F, Emile JF, et al. Dramatic efficacy of vemurafenib in both multisystemic and refractory Erdheim-Chester disease and Langerhans cell histiocytosis harboring the BRAF V600E mutation. Blood 2013;121:1495-1500. 